\title{
Editorial: Stochastic models of manufacturing and service system operations
}

\author{
George Liberopoulos • James MacGregor Smith • \\ Chrissoleon T. Papadopoulos • Tullio Tolio
}

Published online: 19 September 2013

(C) Springer Science+Business Media New York 2013

This volume originates from the Seventh International Conference on "Stochastic Models of Manufacturing and Service Operations", which took place in Ostuni, Italy, in 7-12 June, 2009. The aim of this conference was to serve as a forum for researchers, academics and industrialists to discuss their most recent research findings and to provide them with opportunities for technology transfer. The conference was organized by the Politecnico di Milano, the University of Salento, and ITIA-CNR, Italy. The head of the Organizing Committee was Dr. Tullio Tolio, Professor at the Politecnico di Milano and Director of ITIA-CNR. The conference attracted thirty-three international scholars who presented their work in single-track sessions and many more that participated. Professor Horst Tempelmeier of the University of Cologne, Germany, gave the plenary talk. The previous six biannual conferences of this series were held in Greece (1997-2005) and the Netherlands (2007) under the name "International Conference on Analysis of Manufacturing Systems" and variants of it.

To accomplish a wider dissemination of the results that were reported at the conference, along with those obtained by other researchers working in the area of stochastic modeling

G. Liberopoulos ( $\varangle)$

Department of Mechanical Engineering, University of Thessaly, Pedion Areos, 38334 Volos, Greece e-mail: glib@mie.uth.gr

J. MacGregor Smith

Department of Mechanical and Industrial Engineering, University of Massachusetts Amherst, 111B Marston Hall, 160 Governors Drive, Amherst, MA 01003-2210, USA

e-mail: jmsmith@ecs.umass.edu

C.T. Papadopoulos

Department of Economics, Aristotle University of Thessaloniki, Law School Building, University

Campus, 54124 Thessaloniki, Greece

e-mail: hpap@econ.auth.gr

T. Tolio

Department of Mechanical Engineering, Politecnico di Milano, 1 Via la Masa, 20156 Milan, Italy

e-mail: tullio.tolio@mecc.polimi.it 
of manufacturing and service system operations, an open call for papers in the Annals of Operations Research (AOR) was announced after the conference, leading to this volume. The eleven novel articles published herein were selected among 32 submitted manuscripts, following the standard, rigorous AOR review procedures. They deal with problems in the areas of performance evaluation of production lines with unreliable machines, queueing network analysis of manufacturing systems, analysis and design of production/inventory control policies, assessment of the value of capacity flexibility, stochastic lot scheduling with setups, and system evaluation under cost parameter uncertainty, reliability, and quality control.

In the first paper, Colledani and Gershwin develop a decomposition method for evaluating the performance of a continuous-flow, serial production line with stochastic-capacity machines, modeled by general continuous-time Markov chains, and finite-capacity buffers. The method is based on decomposing the line into several two-machine, one-buffer subsystems and solving each subsystem exactly. They demonstrate numerically the high accuracy of the method.

Gebennini et al. develop an analytical solution for a transfer line with two unreliable machines separated by a finite-capacity buffer, in which the first machine is forced to stop producing when the buffer gets full and is restarted again when the buffer empties, in order to minimize the frequency of stops and restarts, which incur high costs.

Bierbooms et al. develop a decomposition method for evaluating the performance of a single-server tandem queueing network with general service times and finite buffers. The method is based on decomposing the network into single-buffer subsystems and analyzing each subsystem in isolation, taking into account service time dependencies due to blocking. An extensive numerical study shows that this approach produces very accurate performance estimates.

Satyam and Krishnamurthy develop a method for evaluating the performance of a multiproduct manufacturing system with batch-size constraints, operated under CONWIP control. The method uses two-moment approximations to estimate the performance of individual stations, and suitable stochastic transformation equations to relate the arrival and departure processes at different stations. Numerical studies indicate that the proposed method yields fairly accurate performance estimates.

Ioannidis et al. analyze a make-to-stock production system with/without setup costs and delays, perishable inventory, and impatient customers that may balk upon arrival after being notified about delays and renege while waiting during stockouts. They derive analytical expressions for important performance measures and use them to find optimal inventory and admission policies, and investigate the impact of product lifetimes and customer patience times on system performance.

Gurgur develops an effective numerical method to explore and understand the optimal configuration of a multistage, multi-product, decentralized, market-driven production/inventory system that minimizes average inventory holding, subject to a service level constraint through selection of various production and procurement control parameters. She conducts several numerical experiments to understand how the control policies respond to changes in important system parameters.

Buyukkaramikli et al. consider a make-to-order production system with parallel processing units that must meet customer orders with random demand lead times. For this system, they carry out a computational study on the cost savings that can be realized with the use of flexible crews via contractual hiring agreements with an external labor supply agency that can periodically provide an agreed number of crews.

Liberopoulos et al. consider a variant of the stochastic economic lot scheduling problem, in which a machine must produce several grades of a product family to meet random 
demand for each grade. The machine may only change over between neighboring grades and produces continuously at a constant rate even during change-overs. They develop a decomposition-based heuristic for solving the change over decision problem, which they model as an MDP, and report on numerical experimentation with the heuristic.

Zhou and Guan consider a single-item two-stage stochastic lot-sizing problem under cost parameter uncertainty. For this problem, they develop an extended LP formulation in a higher dimensional space that can provide integral solutions, and demonstrate numerically that the extended formulation is more efficient and performs more stable than the two-stage stochastic MIP formulation.

Kharoufeh et al. derive transform expressions for the lifetime distribution of manufacturing equipment that degrades due to its complex operating environment for two different stochastic failure model cases. In the first case, the environment is modeled as a temporally nonhomogeneous continuous-time Markov chain, while in the second it is modeled as a temporally homogeneous semi-Markov process on a finite space.

Finally, Rotondo et al. develop an analytical model for the prediction of the number of unsampled items (indicating quality risk) between consecutive samples at the machines of a non-sampling station of a multiproduct, multistage, parallel processing manufacturing system subject to sequence disorder and multiple stream effects. The development of this model is supported by the analysis of the data generated by a simulation of the system.

In closing, we wish to thank the numerous anonymous referees who gave their valuable time to review the papers submitted to this volume. We also want to thank Professor Endre Boros, Editor-in-Chief of AOR, and Katie D'Agosta, Managing Editor of AOR, for supporting the volume and offering their expert guidance and efforts to make this volume of the Annals a published reality. 